\title{
2. AGES AND KINEMATICS OF CLUSTERS
}

\section{O. J. EGGEN}

\section{California Institute of Technology}

The recent emphasis on stellar evolution, especially as seen in star clusters, has given us - mainly through Sandage's synthesis of the available material - a good outline of the evolutionary process. Although work of a survey nature obviously must be continued, if only to turn up clusters with an age not represented among those now known, I believe the time has come to concentrate a major effort on detailed

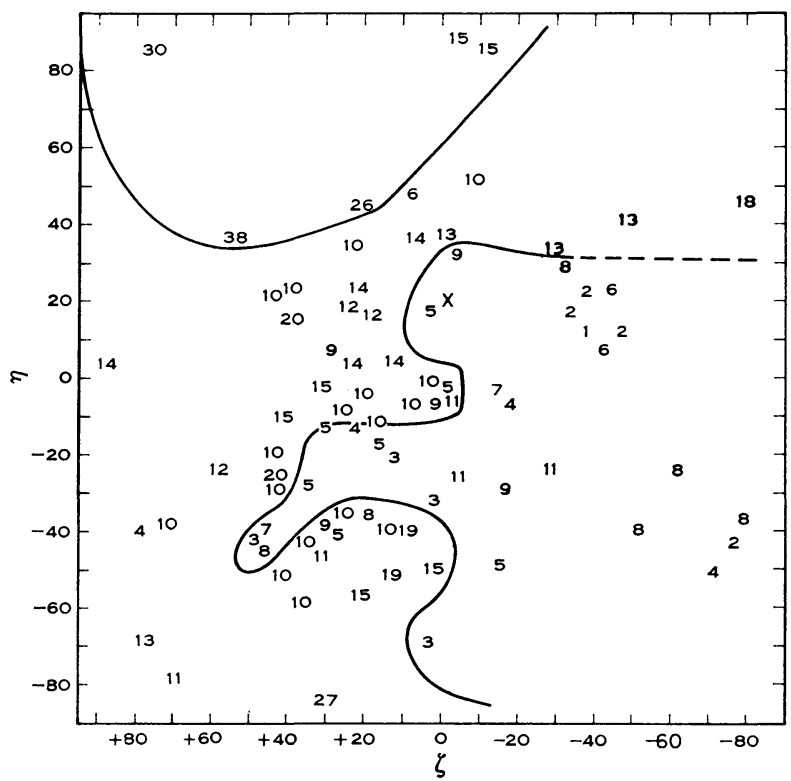

Fig. 1.-The $\eta, \zeta$ (Heckmann and Lübeck 1958) distribution of reddening values $\left(0^{\mathrm{m}} .01\right)$ in the $\alpha$ Persei cluster. The cross represents $\alpha$ Persei itself.

studies of the brighter known clusters of various ages. Our knowledge of even the Hyades and Pleiades clusters is far from complete. A full account cannot be given here but I would like to mention some examples of the details in the colour-magnitude arrays that may eventually lead to important tests, or revisions, of current evolutionary models.

1. An extensive astrometric study of the region of $\alpha$ Persei by Heckmann and Lübeck (1958) gives a rather complete census of cluster members to near visual magnitude 11:5. Mitchell (1960) has obtained three-colour photometric results for all of the known cluster members. The interstellar absorption is not uniform across the cluster field. The values of $E(B-V)$ for the main sequence cluster stars, not known to be binaries, are shown in the $\eta, \zeta$ (Heckmann and Lübeck 1958) diagram of Figure 1. The intrinsic $(U-B, B-V)$-relation used for the B-type stars was slightly modi- 
fied (Eggen 1963a) from that given by Johnson and Morgan (1953). The position of the supergiant $\alpha$ Persei, which is indicated in Figure 1 by a cross, is apparently in a region of relatively low absorption and $E(B-V)$, which is uncertain, is near +0 m05. The corrected colour-magnitude array is shown in Figure 2; the Pleiades main sequence, adjusted for a modulus of $m-M=6.25$ for the $\alpha$ Persei cluster, is shown as a continuous curve. A few stars included as cluster members on the basis of the proper motion but which fall considerably below (crosses) or above (circled crosses) the main sequence are possibly non-members. There is little doubt that the uneven distribution of stars along the main sequence is a real effect. Although the astrometric and photometric results for NGC 1039 (M34) are not as complete,

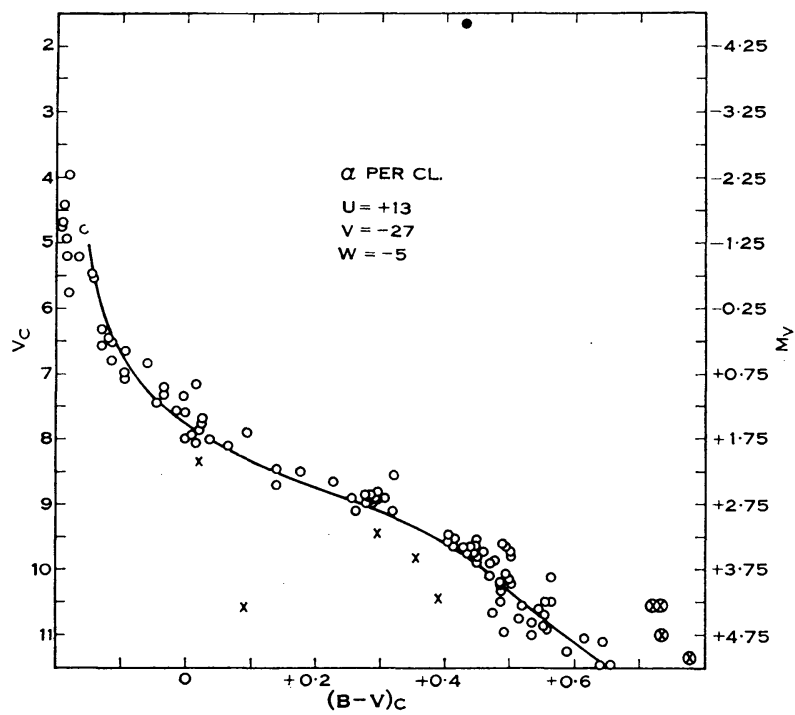

Fig. 2.-The colour-magnitude array, corrected for interstellar absorption, of the $\alpha$ Persei cluster. The continuous curve represents the Pleiades main sequence, adjusted for a modulus of $m-M=6^{\mathrm{m}} .25$ for the $\alpha$ Persei cluster. The supergiant $\alpha$ Persei is indicated by a filled circle.

this cluster shows an almost identical distribution of stars on the main sequence (Johnson 1954, Fig. 1). Especially noteworthy are the gaps at $B-V$ near +0 m 15 and $+0 \mathrm{~m} .35$.

2. Close examination of the galactic clusters older than the Hyades reveals a distinct gap between the evolved and unevolved main sequence stars in the colourmagnitude array. This gap, which is about $0 \mathrm{~m} .5$ wide, is especially noticeable in the rich clusters M67 (Eggen and Sandage 1963) and NGC 2477 (Eggen and Stoy 1961). A recent extensive photoelectric study of NGC 752 (Eggen 1963c) leaves little doubt that this cluster also shows the same phenomena. This gap may be connected with Brownlee's (1963) theoretical result that a solar model with initial $X=0 \cdot 64$ and $Z=$ 0.04 at an age something greater than $5 \times 10^{9}$ years suddenly undergoes a very rapid evolution. Brownlee concluded that if his model was correct, the colour-luminosity 
arrays in clusters near $10^{9}$ years old should be devoid of stars with $M_{V}$ near $+3^{\mathrm{m}}$ to $+4^{\mathrm{m}}$. The observed gap in the clusters mentioned above occurs near the $M_{V}=+2^{\mathrm{m}}$.5.

3. There are several important matters of detail still unsettled in the colourmagnitude arrays of globular clusters. Not the least important of these is the position of the "knee" in the colour-magnitude array (Sandage 1963). Because the available data for the field stars around the brightest globular clusters are insufficient, the corrections for interstellar absorption cannot be disentangled from the blanketing corrections and the exact position of the knee is uncertain in most cases. There is an additional uncertainty in the photometric precision at the light levels involved. For the same reasons the evidence for a spread in the ultraviolet excess among the classical globular clusters is very weak. The details of the brighter parts of the cluster colour-magnitude arrays are obscured by the presence of field stars. Little progress can be made in interpreting this part of the cluster arrays until the type of attack made by Woolley (1963) and his associates on $\omega$ Centauri is extended to other clusters. The astrometric and spectroscopic results obtained by the Herstmonceux group give us for the first time a view of the brightest stars in a globular cluster that is unobscured by field stars. When a similar view is available for several clusters, and the highest quality photometric results are also available for the same clusters, we may be able to test the now current, conflicting theories concerning evolution of these stars.

4. In some clusters that are younger than about $10^{7}$ years, short-period eclipsing stars of the "contact" type (i.e. with $\beta$ Lyrae or W Ursae Majoris type light curves) are found. The known systems of this type occur among the two or three brightest stars in the cluster. The observational evidence is still fragmentary but it does indicate that these contact binaries occur among the bright stars only when T-Tauri stars are rare or absent among the fainter cluster stars. Colour-magnitude arrays of the clusters involved, such as NGC 6871 (with V448 Cygni), NGC 7160 (with EM Cephei), and NGC 6383 (with V701 Scorpii), are given elsewhere (Eggen $1963 d)$.

It is, I think, obvious that future progress in the understanding of stellar evolution depends to a large degree on the clarity with which we can see the kinematical picture. The apparent motions of the nearest bright stars are fairly well known, although there is still an uncertainty a bout the systematic errors in both proper motion and radial velocity systems. However, with the exception of only one or two of the nearest clusters, our present knowledge of the radial velocity and, especially, the proper motion of clusters is extremely limited.

The oldest galactic clusters such as M67 and NGC 188 are quite distant and they contain faint stars for which the proper motions are not well known. The available evidence indicates that $\mathrm{M} 67$ has $(U, V)$ near $(+50,-30)$, which is similar to $(+50,-45)$ for the $\zeta$ Herculis group. The colour-magnitude arrays are nearly identical and it is well within the uncertainty of the $(U, V)$ values for the cluster that the space motions of the cluster group are identical.

The space motion of the Pleiades is as well determined as any known for the younger clusters. The values for the brightest cluster stars (Eggen 1961), based on a modulus of $m-M=5^{\mathrm{m}} \cdot 55$, are: 


\begin{tabular}{ccccccc} 
Star & $\mu(\alpha)$ & $\mu(\delta)$ & $\rho$ & $U$ & $V$ & $W$ \\
17 Tau & +0.0013 & $-0^{\prime} 043$ & $+12 \cdot 4$ & +13 & -25 & -16 \\
19 Tau & $+0 \cdot 0014$ & -0.042 & $+5 \cdot 5$ & +7 & -26 & -13 \\
20 Tau & +0.0015 & -0.043 & $+7 \cdot 5$ & +9 & -27 & -13 \\
23 Tau & +0.0016 & -0.042 & $+6 \cdot 2$ & +8 & -27 & -12 \\
27 Tau & +0.0016 & -0.043 & $+10 \cdot 1$ & +12 & -27 & -14 \\
\cline { 3 - 7 } & & & $+8 \cdot 3$ & +10 & -27 & -13
\end{tabular}

At first sight the stars appear to have a greater mobility in $U$ than in $V$ but this is almost certainly a reflection of the uncertainty in the radial velocities. The direction cosines for the Pleiades stars are:

$\begin{array}{cccc}\mu(\alpha) & \mu(\delta) & \rho & \\ +44 & +13 & +89 & U \\ -66 & +72 & +22 & V \\ +61 & +68 & -40 & W\end{array}$

and the radial velocity is almost entirely a reflection of the $U$-velocity. The nearly zero dispersion in $V$ results from the near equality of the proper motions, and the real dispersion in $U$ is probably as small. Pearce (Petrie 1963) finds a mean of +7 $\mathrm{km} / \mathrm{sec}$ for the radial velocity of 66 cluster members so that a better value of $(U$, $V, W)$ is probably $(+9,-27,-13)$.

The reasonably reliable material for the $\alpha$ Persei cluster and for NGC 2516, and the much more uncertain results for two other young clusters, give space motions that, within the uncertainty of the fundamental data, are nearly identical with that of the Pleiades:

$\begin{array}{lcclc}\quad \text { Cluster } & \text { Dist. (pc) } & U & V & W \\ \alpha \text { Per } & 175 & +13 & -27 & -5 \\ \text { NGC } 2516 & 365 & +10 & -24 & +4 \\ \text { IC } 2602 & 155 & +4: & -25: & -3: \\ \text { NGC } 1039 & 440 & +3: & -27: & -20:\end{array}$

The very similar colour-magnitude arrays for the $\alpha$ Persei cluster and NGC 1039 are discussed above. The colour-magnitude arrays for both NGC 2516 and IC 2602 are particularly interesting because of their great similarity with that of the Pleiades cluster; all three clusters show a decided break in the star distribution between $M_{V}=-1^{\mathrm{m}}$ and -0 m5 (Eggen 1963d). Also, both NGC 2516 and IC 2602 have one Ap star at the top of the main sequence $\left(M_{V}=-0^{\mathrm{m}} 5\right)$, below the break mentioned above; the shell star Pleione falls on the colour-magnitude array of the Pleiades at the same luminosity. The possibility that the few stars brighter than $M_{V}$ near $-1^{\mathrm{m}}$ in the Pleiades, NGC 2516, and IC 2602 represent a later generation than the bulk of the "older" cluster members, which may have an age near $5 \times 10^{8}$ years, is discussed elsewhere (Eggen 1963b,d; also Herbig 1963). This possibility would be consistent with the fact that several red giants in the solar neighbourhood appear to share the motion of the Pleiades; a few examples are the following:

\begin{tabular}{|c|c|c|c|c|c|c|c|c|}
\hline$H R$ & $V_{E}$ & $B-V$ & $\mathrm{~S}$ & p & $M_{V}$ & $U$ & $V$ & $W$ \\
\hline 2113 & $4^{\mathrm{m}_{50}}$ & $+1^{\mathrm{m}_{23}}$ & $\mathrm{~K} 2$ & III & $+0^{\mathrm{m}} \cdot 70$ & +9 & -27 & -12 \\
\hline 2462 & $4 \cdot 91$ & $+0 \cdot 88$ & gG8 & & $+0 \cdot 70$ & +8 & -26 & -7 \\
\hline 3080 & $3 \cdot 70$ & $+1 \cdot 02$ & G5 & III & $+0 \cdot 70$ & +8 & -24 & -4 \\
\hline 3709 & $4 \cdot 84$ & +0.91 & G8 & III & $+0 \cdot 70$ & +10 & -26 & +1 \\
\hline 4737 & $4 \cdot 35$ & $+1 \cdot 13$ & $\mathrm{Kl}$ & III & $+0 \cdot 25$ & +8 & -27 & +1 \\
\hline
\end{tabular}


Of equal interest is the fact that many younger stars $\left(10^{7}\right.$ years $)$ in the solar neighbourhood also appear to share the motion of the Pleiades. The stars in Table 1 represent about $33 \%$ of those within $250 \mathrm{pc}$ of the Sun with spectral type earlier than B7, brighter than visual magnitude $5 \cdot 0$, and for which well-determined apparent

TABLE 1

YOUNG STARS WITHIN 250 PC OF THE SUN AND SPACE MOTION SIMILAR TO THAT OF THE PLEIADES

\begin{tabular}{|c|c|c|c|c|c|c|c|c|c|}
\hline Name & $V_{E}$ & $B-V$ & $E$ & $U$ & $V$ & $W$ & $m-M$ & $M_{V}$ & $\mathrm{Sp}$ \\
\hline $\begin{array}{l}\epsilon \text { Per } \\
\beta \text { Cru } \\
\alpha \text { Vir }\end{array}$ & $\begin{array}{l}2 \cdot 88 \\
1 \cdot 24 \\
0 \cdot 98\end{array}$ & $\begin{array}{c}m \\
-0 \cdot 17 \\
-0 \cdot 17 \\
-0 \cdot 24\end{array}$ & $\begin{array}{r}\mathrm{m} \\
+0.15 \\
+0.04 \\
0.00\end{array}$ & $\begin{array}{l}+13 \\
+\quad 4 \\
+10\end{array}$ & $\begin{array}{l}-27 \\
-27 \\
-27\end{array}$ & $\begin{array}{l}-7 \\
-8 \\
-8\end{array}$ & $\begin{array}{l}6 \cdot 35 \\
5 \cdot 25 \\
5 \cdot 55\end{array}$ & $\begin{array}{l}-3 \cdot 9 \\
-4 \cdot 1 \\
-3 \cdot 9\end{array}$ & $\begin{array}{l}\mathrm{B} 0.5 \mathrm{~V} \\
\mathrm{~B} 0.5 \mathrm{~V} \\
\mathrm{~B} 1 \mathrm{~V}\end{array}$ \\
\hline . & \multicolumn{3}{|c|}{$(B-V)_{0}=-0 \cdot 26$} & +9 & -27 & -8 & & $-4 \cdot 0$ & $\mathrm{~B} 0 \cdot 5 \mathrm{~V}(-3 \cdot 6)$ \\
\hline $\begin{array}{l}\text { HR } 6247 \\
o \text { Cas } \\
v \text { Cen } \\
\beta \text { Lup }\end{array}$ & $\begin{array}{l}3 \cdot 0 \mathrm{v} \\
4 \cdot 60 \\
3 \cdot 40 \\
2 \cdot 66\end{array}$ & $\begin{array}{l}-0.23 \\
-0.07 \\
-0.25 \\
-0.22\end{array}$ & $\begin{array}{r}+0.02 \\
+0.11 \\
0.00 \\
+0.02\end{array}$ & $\begin{array}{l}+4 \\
+8 \\
+10 \\
+15\end{array}$ & $\begin{array}{l}-27 \\
-27 \\
-27 \\
-27\end{array}$ & $\begin{array}{l}-7 \\
-5 \\
-7 \\
-10\end{array}$ & $\begin{array}{l}7 \cdot 00 \\
7 \cdot 00 \\
6 \cdot 30 \\
5 \cdot 80\end{array}$ & $\begin{array}{l}-3 \cdot 2 \\
-2 \cdot 7 \\
-2 \cdot 9 \\
-3 \cdot 2\end{array}$ & $\begin{array}{l}\mathrm{B} 1 \cdot 5 \mathrm{~V} \\
\mathrm{~B} 2 \mathrm{~V} \\
\mathrm{~B} 2 \mathrm{~V} \\
\mathrm{~B} 2 \mathrm{~V}\end{array}$ \\
\hline$\beta$ Lup & \multicolumn{3}{|c|}{$(B-V)_{0}=-0 \cdot 23$} & +9 & -27 & -7 & & $-3 \cdot 0$ & $\mathrm{~B} 2 \mathrm{~V}(-2 \cdot 6)$ \\
\hline$\lambda \mathrm{Cru}$ & $4 \cdot 62$ & $-0 \cdot 15$ & $+0 \cdot 04$ & +9 & -27 & -21 & $6 \cdot 75$ & $-2 \cdot 2$ & B3 V \\
\hline$\eta$ Aur & $3 \cdot 16$ & $-0 \cdot 17$ & +0.03 & +14 & -27 & -8 & $4 \cdot 70$ & $-1 \cdot 6$ & B3 V \\
\hline$x$ Cen & $4 \cdot 35$ & $-0 \cdot 20$ & $0 \cdot 00$ & +7 & -27 & -5 & $6 \cdot 45$ & $-2 \cdot 1$ & B3 V \\
\hline HR 5967 & $4 \cdot 90$ & $-0 \cdot 14$ & $+0 \cdot 04$ & +8 & -27 & -12 & $6 \cdot 55$ & $-1 \cdot 8$ & B3 V \\
\hline$\mu \mathrm{Tau}$ & $4 \cdot 30$ & -0.08 & $+0 \cdot 10$ & +15 & -27 & -3 & $6 \cdot 00$ & $-2 \cdot 0$ & B3 V \\
\hline$\alpha$ Ara & $2 \cdot 91$ & $-0 \cdot 18$ & +0.02 & +12 & -27 & -6 & $4 \cdot 90$ & $-2 \cdot 0$ & B3 V \\
\hline$\alpha \mathrm{Tel}$ & $3 \cdot 50$ & $-0 \cdot 18$ & +0.01 & +8 & -27 & -7 & $5 \cdot 70$ & $-2 \cdot 2$ & B3 V \\
\hline$\alpha \mathrm{Pav}$ & $1 \cdot 91$ & $-0 \cdot 22$ & 0.00 & +10 & -27 & -4 & $4 \cdot 20$ & $-2 \cdot 3$ & B3 V \\
\hline \multirow[t]{2}{*}{ HR 5471} & $4 \cdot 00$ & $-0 \cdot 18$ & +0.02 & +9 & -27 & -10 & $6 \cdot 20$ & $-2 \cdot 3$ & B3 V \\
\hline & \multicolumn{3}{|c|}{$(B-V)_{0}=-0 \cdot 20$} & +10 & -27 & -8 & & $-2 \cdot 0$ & B3 V $(-2 \cdot 0)$ \\
\hline$\alpha$ Eri & 0.50 & $-0 \cdot 16$ & $0 \cdot 00$ & +8 & -27 & -6 & $3 \cdot 30$ & $-1 \cdot 8$ & B5 V \\
\hline$\kappa$ Нуа & $4 \cdot 98$ & $-0 \cdot 15$ & +0.02 & +12 & -27 & -25 & $6 \cdot 75$ & $-1 \cdot 8$ & B5 V \\
\hline o Lup & $4 \cdot 32$ & $-0 \cdot 16$ & +0.02 & +9 & -27 & -5 & $6 \cdot 30$ & $-2 \cdot 0$ & B5 V \\
\hline$\iota \mathrm{Aql}$ & $4 \cdot 33$ & -0.08 & +0.09 & +10 & -27 & -6 & $5 \cdot 90$ & $-1 \cdot 8$ & B5 III \\
\hline$\theta \mathrm{CrB}$ & $4 \cdot 22$ & $-0 \cdot 13$ & +0.05 & +10 & -27 & -9 & $6 \cdot 00$ & $-1 \cdot 9$ & B5 nn \\
\hline \multirow[t]{2}{*}{$\alpha$ Gru } & $1 \cdot 71$ & $-0 \cdot 14$ & +0.01 & +9 & -27 & -16 & $2 \cdot 55$ & $(-0 \cdot 9)$ & B5 V \\
\hline & \multicolumn{3}{|c|}{$(B-V)_{0}=-0 \cdot 17$} & +10 & -27 & -10 & & $-1 \cdot 9$ & B5 $\mathrm{V}(-1 \cdot 3)$ \\
\hline$\alpha \mathrm{Col}$ & $2 \cdot 64$ & $-0 \cdot 13$ & +0.02 & +12 & -28 & -18 & $3 \cdot 00$ & $-0 \cdot 4$ & B8 V $(-0.5)$ \\
\hline$\alpha \operatorname{Lup}$ & $2 \cdot 30$ & $-0 \cdot 21$ & +0.04 & +10 & -27 & -6 & $7 \cdot 00$ & $-4 \cdot 8$ & B1 III $(-4 \cdot 3)$ \\
\hline$\delta$ Per & $3 \cdot 02$ & $-0 \cdot 13$ & $+0 \cdot 03$ & +5 & -27 & -4 & $5 \cdot 40$ & $-2 \cdot 5$ & B5 III \\
\hline HR 4618 & $4: 46$ & $-0 \cdot 17$ & $+0 \cdot 03$ & +12 & -27 & -12 & $6 \cdot 70$ & $-2 \cdot 3$ & B6 III \\
\hline HR 5378 & $4 \cdot 40$ & $-0 \cdot 19$ & +0.03 & +7 & -27 & -6 & $6 \cdot 30$ & $-2 \cdot 0$ & B6 III \\
\hline \multirow[t]{2}{*}{ o Lup } & $4 \cdot 31$ & $-0 \cdot 16$ & $+0 \cdot 03$ & +8 & -27 & -5 & $6 \cdot 30$ & $-2 \cdot 1$ & B6 III \\
\hline & \multicolumn{3}{|c|}{$(B-V)_{0}=-0 \cdot 19$} & +8 & -27 & -7 & & $-2 \cdot 2$ & B6 III $(-3 \cdot 0)$ \\
\hline
\end{tabular}


motions are available (Eggen 1961); the values of $M_{V}$ given in parentheses in the last column of the table are the mean values assigned to the spectral type and luminosity class by Keenan and Morgan (1951). The individual luminosities were obtained by assuming that $V=-27 \mathrm{~km} / \mathrm{sec}$ but introducing a range in $V$ that is similar to the one shown in $U(10 \mathrm{~km} / \mathrm{sec})$ would result in only \pm 0 m.3 dispersion in $M_{V}$ for a given spectral type. In other words, the present range in both $U$ and $V$ for these stars may be near $10 \mathrm{~km} / \mathrm{sec}$ with a mean $(U, V)$ near $(+9,-27)$. If so the relative positions have not changed by more than about $100 \mathrm{pc}$ since formation. However, in $10^{8}$ years an equal volume of space, also containing the Pleiades, will contain only those stars in Table 1 that now have true values of $V$ within 1 or $2 \mathrm{~km} / \mathrm{sec}$ of $-27 \mathrm{~km} / \mathrm{sec}$. Since approximately $30 \%$ of the bluest Ap stars ( $\lambda 4200, \mathrm{Si}, \mathrm{Mn}$ ) near the Sun, which have $B-V$ colours similar to normal B5 to B7 stars, are also moving with the same space motion as the Pleiades cluster (Eggen 1963b), it appears that approximately a third of all the youngest stars in the solar vicinity may belong to a Pleiades group. It is remarkable that Table 1, plus $\beta$ Tauri (B7 III), which should perhaps be considered as an outrider to the Pleiades with $m-M=2$ m85 and $(U, V, W)=(+8$, $-28,-15$ ), includes 4 out of the 5 ( $\alpha$ Leonis excepted) B-type stars within 50 pc of the Sun.

The possibility that a very large percentage of the youngest population near the Sun - perhaps the whole Gould Belt complex - was produced from one gas cloud has to be taken into account when interpreting the solar motion with respect to these young stars. The mean motion of this complex with respect to the Sun is sensitive to the distance scale (i.e. to the luminosity calibration of the B stars) adopted, but $\bar{U}$ is probably not less than $10 \mathrm{~km} / \mathrm{sec}$ and $\bar{V}$ not less than $-25 \mathrm{~km} / \mathrm{sec}$. These mean values may reflect the motion of the parent cloud and if this is moving in a circular galactic orbit the resulting solar motion with respect to this circular velocity is considerably higher than that given by the so-called "basic" solar motion of $(U, V)=(+10,-10)$.

\section{References}

Brownlee, R. (1963).-(Herstmonceux Conference) Royal Obs. Bull. (in press).

EgGen, O. J. (1961).-Royal Obs. Bull. No. 41.

Eggen, O. J. $(1963 a)$. - Ap. J. Supp. No. 76.

EgGen, O. J. (1963b)._- "Stars and Stellar Systems." (General Ed. G. P. Kuiper.) (Univ. Chicago Press: in press).

EgGen, O. J. (1963c).-Ap. J. (in press).

EgGen, O. J. (1963d).-(Herstmonceux Conference) Royal Obs. Bull. (in press).

EggeN, O. J., and Sandage, A. R. (1963).-(in press).

Eggen, O. J., and Stoy, R. H. (1961).-Royal Obs. Bull. No. 24.

HeckmanN, O., and Lubeck, K. (1958).-Zs. f. Ap. 45: 243-63.

Herbig, G. H. (1963).-(Herstmonceux Conference) Royal Obs. Bull. (in press).

Johnson, H. L. (1954).-Ap. J. 119: 185-7.

Johnson, H. L., and Morgan, W. W. (1953).-Ap. J. 117 : 313-52.

Keenan, P. C., and Morgan, W. W. (1951)._-"Astrophysics." (Ed. J. A. Hynek.) pp. 12-25. (McGraw-Hill Book Co. Inc.: New York.)

Mttchell, R. I. (1960).-Ap. J. 132: 68-75.

Petrie, R. M. (1963).-(Herstmonceux Conference) Royal Obs. Bull. (in press).

Sandage, A. R. (1963).--(Herstmonceux Conference) Royal Obs. Bull. (in press).

Woolley, R. v. D. R. (1963).--(Herstmonceux Conference) Royal Obs. Bull. (in press). 


\title{
Discussion
}

Buscombe: Why was IC 2602 selected if its radial velocity is not well known? Two other nearby southern clusters, IC 2391 and M7, contain rather many peculiar A stars.

Eggen: Proper motions are not well known for the last two clusters.

Blaauw: In determining distances and luminosities, Dr. Eggen assumed a constant value of the velocity component $V$ for the stars involved. This was justified by the introductory remarks about the natural selection of stars of ages of $5 \times 10^{8} \mathrm{yr}$ in the solar neighbourhood, these stars having been formed in a region $<500$ pc. What justification is there in applying this theory to the $\mathrm{B}$ stars which are 10 times younger?

Eggen: The point is that at least those with $V=-27$ are members of the association; others may also belong but because of the dispersion in $V$ they will not be picked up.

\section{SPACE DISTRIBUTION OF 156 GALACTIC STAR CLUSTERS AND OF 61 HII REGIONS*}

\author{
W. BECKER
}

Astronomische Anstalt, Basel-Binningen

The galactic star clusters are today the only objects whose individual distances and ages can be determined rather precisely. Therefore the space distribution as a function of time can be studied. In case they form a spiral structure, the lifetime of such a structure can be studied too.

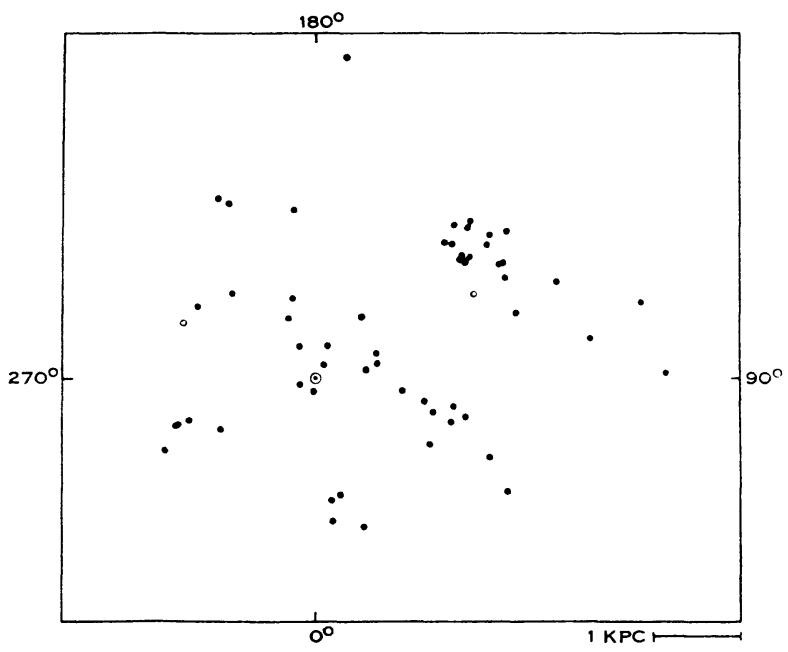

Fig. 1.-Distribution of clusters with earliest spectral type between $\mathrm{O}$ and $\mathrm{B2}$.

In the following, we rely exclusively on distances obtained by the method of three-colour photometry. As this method is used in two different ways, all distances are reduced to a homogeneous system applying the method developed at Basel-

* Published in detail in Zs. f. Ap. 56: 257-63 and 57: 117-34. 